\title{
COMMENT
}

\section{Another dimension: investigations of molecular magnetism using muon-spin relaxation}

\author{
Tom Lancaster \\ Durham University, Department of Physics, South Road, Durham, DH1 3LE UK \\ E-mail: tom.lancaster@durham.ac.uk
}

Stephen J Blundell

University of Oxford, Clarendon Laboratory, Parks Road, Oxford, OX1 3PU, UK

Francis L Pratt

STFC ISIS Facility, Rutherford Appleton Laboratory, Chilton Didcot, OX11 0QX, UK

\begin{abstract}
We review examples of muon-spin relaxation measurements on moleculebased magnetic coordination polymers, classified by their magnetic dimensionality. These include the one-dimensional $s=1 / 2$ spin chain $\mathrm{Cu}(\mathrm{pyz})\left(\mathrm{NO}_{3}\right)_{2}$ and the twodimensional $s=1 / 2$ layered material $\left[\mathrm{Cu}\left(\mathrm{HF}_{2}\right)(\mathrm{pyz})_{2}\right] \mathrm{BF}_{4}$. We also describe some of the more exotic ground states that may become accessible in the future given the ability to tune the interaction strengths of our materials through crystal engineering.
\end{abstract}

PACS numbers: 76.75.+i, 75.50.Xx, 75.10.Jm, 75.50.Ee 


\section{Molecule-based magnets}

Molecule-based magnets are systems whose principal structural building blocks are organic molecules [1, 2]. We understand the microscopic origin of the magnetism that arises from such materials in terms of paramagnetic spin centres linked via superexchange pathways. The magnetic centres themselves may be atoms (such as $s=1 / 2 \mathrm{Cu}^{2+}$ ) or uncompensated spins on the molecules (as in the case of radical magnets) and the superexchange pathways will frequently pass through the molecular groups. The great promise of choosing to make our magnets out of molecules is tunability, that is, the possibility of manipulating the carbon chemistry of the molecules to form structures which promote magnetic exchange in certain geometries and to manipulate the strength of the coupling between the magnetic units.

Research on molecular magnetic systems relies on a confluence of experimental and theoretical physics and chemistry. Chemistry is vital in the synthesis of new materials with the desired structural properties which incorporate the necessary magnetic ingredients; experiment allows us to probe the magnetism and we hope that theory can explain it. In this Comment we will discuss some examples of the use and successes of muon-spin relaxation $\left(\mu^{+} \mathrm{SR}\right)$ [3] as an experimental technique in this area. We will classify the systems that we discuss in terms of their magnetic dimensionality, that is, the number of dimensions in which the coupling between magnetic units is strong. The explanation of the physics of such systems is usually given in terms of low-energy models of exchange-coupled spins [4, 5, 6] whose long-wavelength properties have been elucidated using field-theoretic techniques and, in particular, the renormalization group (RG) [7, 8]. In presenting some possible future directions for muon spectroscopy in this field we will describe some of the theoretical models which are currently motivating the synthesis of new materials.

The subject of molecular magnetism has been reviewed thoroughly elsewhere [1, 2], as has early, and more recent, work using $\mu^{+} \mathrm{SR}$ [9, 10, 11, 12, 13. However, it is worth mentioning some of the notable themes from the early muon work, which include: (i) the observation of long-range magnetic order (LRO), such as in the purely organic material $\beta$-phase $p$-NPNN, where oscillations in the muon asymmetry are found below $0.7 \mathrm{~K}[14$; (ii) the observation of dynamics in molecular nanomagnets such as the single molecule magnet $\mathrm{Mn}_{12} \mathrm{Ac}$ [15] and (iii) the possibility of observing collective magnetic behaviour such as the spin-crossover transition [9]. These investigations play to the strengths of $\mu^{+} \mathrm{SR}$ in that they require a probe sensitive to small magnetic moments and their dynamics and the possibility of distinguishing between uniform magnetic effects and minority, strongly magnetic phases.

The Comment is structured as follows: in section 2 we will discuss the theoretical prediction and realization of one-dimensional magnetism from molecular chains. In section 3 we turn to layered two-dimensional systems and in section 4 to the recently realised possibility of switching between dimensionalities. Finally we discuss some of the possible future directions for this field in section 5 . 


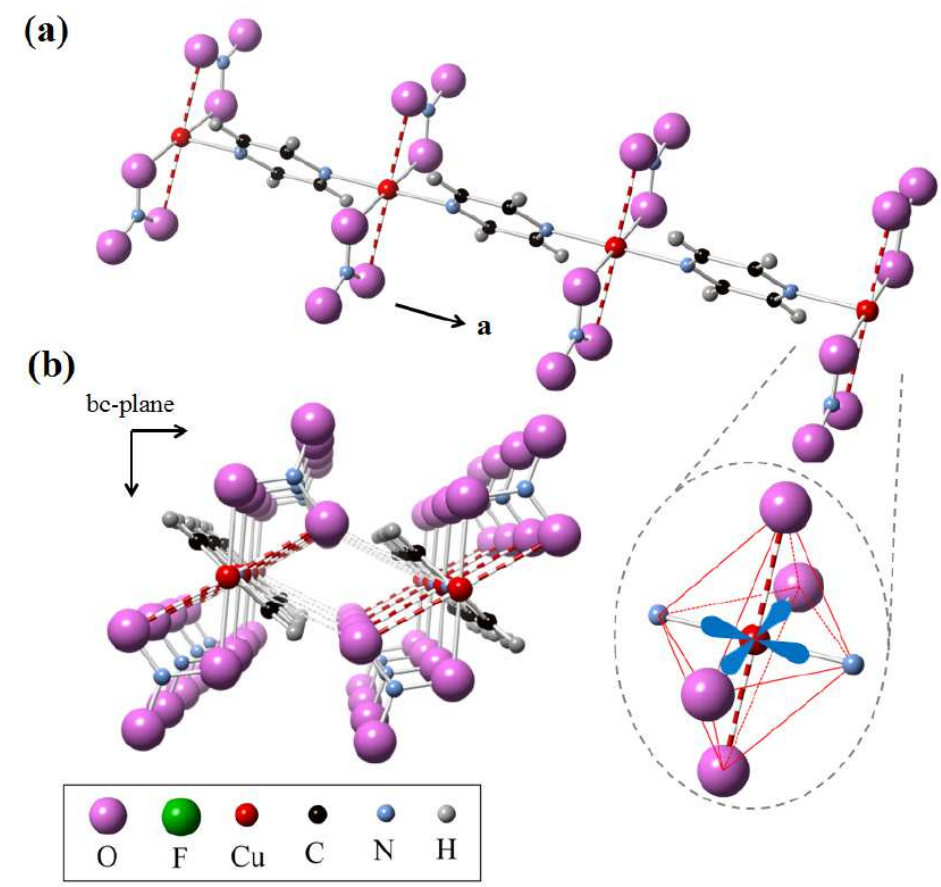

Figure 1. The structure of $\mathrm{Cu}(\mathrm{pyz})\left(\mathrm{NO}_{3}\right)_{2}$. (a) The Cu-pyz-Cu chains. (b) The chain packing viewed along the $a$-direction. Figure taken from Ref. [20].

\section{Magnetism in one dimension}

Much recent work on molecular magnets using $\mu^{+}$SR has concerned materials whose low-energy physics may be thought of as arising from interactions which are constrained to act in less than three spatial dimensions. Such physics usually achieved by forming an array of transition metal ions, such as $s=1 / 2 \mathrm{Cu}^{2+}$ and linking these using one or more molecular ligands in such a way as to promote strong interactions between the magnetic ions only along particular directions and weaker ones along others.

As an example, we will consider a material whose structure promotes strong, antiferromagnetic superexchange along one-dimensional, parallel, linear chains, and weaker exchange in the other two perpendicular directions. Such a material may be constructed if we link $\mathrm{Cu}^{2+}$ ions along one direction using pyrazine (pyz $\equiv \mathrm{C}_{4} \mathrm{H}_{4} \mathrm{~N}_{2}$ ) ligands and separate the chains using $\mathrm{NO}_{3}$ groups. The resulting material formed from this recipe is $\mathrm{Cu}\left(\right.$ pyz) $\left(\mathrm{NO}_{3}\right)_{2}$ and is shown in figure 1 .

To understand the physics of such materials we seek to describe them using simple spin models. To a good first approximation the crystal field surrounding the $\mathrm{Cu}^{2+}$ ions does not cause the spin to favour any particular spatial direction, resulting in a socalled Heisenberg spin [16]. The resulting one-dimensional $(d=1)$ quantum Heisenberg antiferromagnet (1DQHAF) may be described by a model Hamiltonian [4, 5, 17]

$$
\hat{H}=J \sum_{\langle i j\rangle} \hat{\boldsymbol{S}}_{i} \cdot \hat{\boldsymbol{S}}_{j},
$$




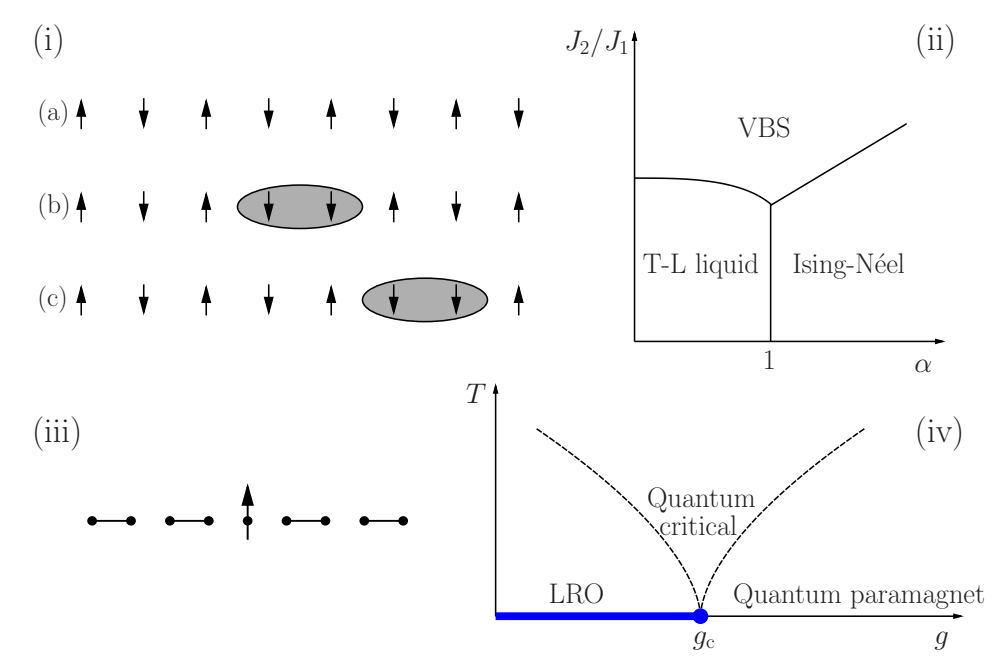

Figure 2. (i) The spinon excitation in a $d=1$ chain. (ii) The phase diagram of a $d=1$ chain system with nearest neighbour coupling $J_{1}$, next-nearest neighbour coupling $J_{2}$ and anisotropy parameter $\alpha$ [VBS=valence bond solid; T-L liquid=Tomonaga Luttinger liquid (see Section 5).] (iii) The valence bond solid with a single $s=1 / 2$ excitation. (iv) The phase diagram of a $d=2$ system showing a quantum critical point at a coupling $g_{\mathrm{c}}$ and a quantum critical region at elevated temperatures.

where $i$ and $j$ label spins along the same chain and the sum is over unique nearest neighbours bonds only. For materials such as $\mathrm{Cu}($ pyz $)\left(\mathrm{NO}_{3}\right)_{2}$ the parameter $J$ may be determined from the result of magnetic susceptibility or pulsed field magnetization (see below).

The model Hamiltonian in eqn 1 is very interesting and has motivated a wide range of research [4, 5]. It possesses a continuous $O(3)$ symmetry (here the global rotation of all of the spins through an arbitrary angle). In common with other $d=1$ models with finite range interactions there will be no LRO for $T>0$. (For $d=1$ this is easy to see: pass the message "line-up" along the chain in the presence of a defect (or a fluctuation) and the message is lost at the weak link: since each spin is only able to communicate with its neighbours the message cannot pass around the weak link). Although this problem may be solved exactly using the famous Bethe ansatz [6] the solution is rather unwieldy and many of our insights arise from the predictions of a continuum model which, as long as the long-range spin-spin coupling is not too large (see section 5 ), results from mapping the problem onto a $d=1$ model of spinless fermions and then using a technique known as bosonization. The predicted state is known as a Tomonaga-Luttinger (T-L) liquid [5, 6] and its excitation spectrum is one of gapless, linearly dispersing, particlelike excitations. Some physical insight into the nature of these excitations comes from figure 2(i). An antiferromagnetically correlated region [as shown in (a)] may be excited if we flip a section of the spins, as has been done after the fourth spin in (b). This creates a spin $1 / 2$ excitation, known as a spinon [shown shaded in figure 2(i)], which may move by hopping by two lattice spacings at a time [shown in (c)]. 


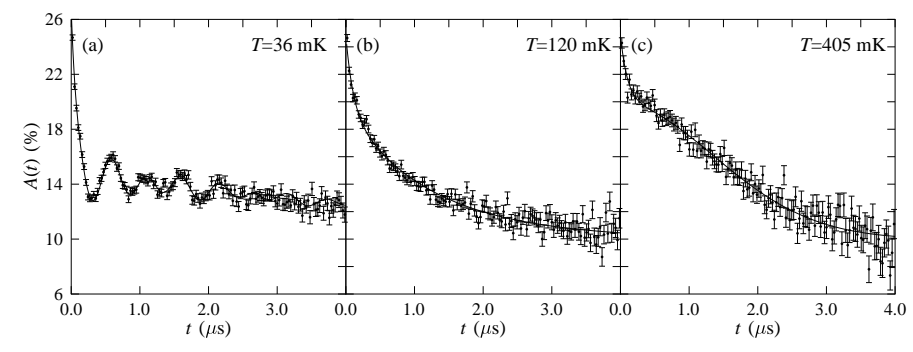

Figure 3. Zero field muon-spin relaxation data measured on $\mathrm{Cu}(\mathrm{pyz})\left(\mathrm{NO}_{3}\right)_{2}$, showing long-range magnetic order below $T_{\mathrm{N}}=107 \mathrm{mK}$ [21]. .

The idealisation that leads to the T-L model has ignored many interactions in $\mathrm{Cu}($ pyz $)\left(\mathrm{NO}_{3}\right)_{2}$ and one of these proves particularly relevant: the interaction between chains parametrised by an exchange interaction $J_{\perp}$. This changes the Hamiltonian to

$$
\hat{H}=J \sum_{\langle i j\rangle} \hat{\boldsymbol{S}}_{i} \cdot \hat{\boldsymbol{S}}_{j}+J_{\perp} \sum_{\left\langle i j^{\prime}\right\rangle} \hat{\boldsymbol{S}}_{i} \cdot \hat{\boldsymbol{S}}_{j^{\prime}},
$$

where $i$ and $j^{\prime}$ label spins on adjacent chains. In order to probe $d=1$ behavior a system described by this model we would need to work in a temperature regime where $J_{\perp} \ll T \ll J$. Once we start to approach a regime where the characteristic energy of the thermal fluctuations allows detail on the level of $J_{\perp}$ to be resolved, we should expect that the three-dimensional nature of the material becomes evident and it will magnetically order. However, the strongly anisotropic nature of the exchange $\left(J_{\perp} / J \ll 1\right)$ will have consequences on the ordered state that arises [18] and the ordering temperature $T_{\mathrm{N}}$ is driven downwards by the one-dimensional nature of the system compared to a threedimensional one. The ordered magnetic moments will also be renormalized, making them potentially very small, and difficult to observe with magnetic susceptibility. Finite, but possibly very long, correlation lengths may exist above the ordering temperature, reducing the entropy in the system. Consequently, the entropy change on ordering will be much reduced compared to a more isotropic system and may prevent specific heat measurements from detecting a transition. Add the difficulty of deuterating all of the hydrogen-containing groups in the system (and the possibility of deuteration changing the delicate superexchange pathways [19]) and neutron diffraction also becomes difficult. It is here that $\mu^{+} \mathrm{SR}$ has proven very effective in detecting magnetic transitions in reduced dimensions that have proven difficult to observe using more conventional experimental methods. Before the $\mu^{+} \mathrm{SR}$ study, no magnetic order had been observed in $\mathrm{Cu}(\mathrm{pyz})\left(\mathrm{NO}_{3}\right)_{2}$ despite specific heat measurements made down to $70 \mathrm{mK}$. However, the clear zero-field precession signal from $\mu^{+} \mathrm{SR}$ [21] shows unambiguous evidence for LRO below a transition temperature of $T_{\mathrm{N}}=0.107 \mathrm{~K}$ as shown in figure 3 .

A useful figure of merit in comparing the success with which a material realizes one-dimensionality is $T_{\mathrm{N}} / J$, since this quantity should be zero in the ideal case and of order and close to unity for an isotropic material. This quantity may also be used 
to estimate $J_{\perp}$, which is the parameter of interest in the Hamiltonian in eqn 2. One method of doing this is has been developed from Quantum Monte Carlo computations [22], whose results may be approximated using the expression

$$
\left|J_{\perp}\right| / k_{\mathrm{B}}=\frac{T_{\mathrm{N}}}{4 c \sqrt{\ln \left(\frac{a|J|}{\mathrm{k}_{\mathrm{B}} T_{\mathrm{N}}}\right)+\frac{1}{2} \ln \ln \left(\frac{a|J|}{\mathrm{k}_{\mathrm{B}} T_{\mathrm{N}}}\right)}},
$$

with $a=2.6$ and $c=0.233$. This yields a value of $\left|J_{\perp} / J\right|=4.4 \times 10^{-3}$ for $\mathrm{Cu}$ (pyz) $\left(\mathrm{NO}_{3}\right)_{2}$, demonstrating that it is indeed a very well isolated approximation of a $d=1$ magnet. In addition, coupled chain mean field theory [23] predicts an ordered moment of $0.05 \mu_{\mathrm{B}}$.

The application of a magnetic field to our $d=1$ system acts to polarize the spins. If we imagine doing this at $T=0$ then there will be a point at a field $\mu_{\mathrm{B}} B \gg J$ where all of the spins will be aligned. This aligned state is separated from the partially polarised antiferromagnet by a quantum critical point (QCP) [5] at a field $B_{\mathrm{c}}=2 \mathrm{~J} / \mathrm{g} \mu_{\mathrm{B}}$ (we neglect interchain interactions here). One advantage of investigating molecular magnetic systems such as these is that the exchange strength, and hence the QCP, is typically of order $J \approx 10 \mathrm{~K}$, translating as $B_{\mathrm{c}} \approx 10 \mathrm{~T}$. Although such fields are currently outside the range accessible via $\mu^{+} \mathrm{SR}$ they are accessible using pulsed magnetic fields and the combination of high-field magnetization and muon spectroscopy [24] has proved valuable in fully characterising new materials in recent years. These characteristic energy scales are orders of magnitude smaller than the corresponding ones for inorganic spin chains such as $\mathrm{Sr}_{2} \mathrm{CuO}_{3}$, whose QCP occurs at an experimentally inaccessible field around 200 times larger than that of $\mathrm{Cu}(\mathrm{pyz})\left(\mathrm{NO}_{3}\right)_{2}$.

An even more ideal example of this physics is found in the radical system DEOCC$\mathrm{TCNQF}_{4}$ [25]. In this case no LRO transition is observed using $\mu^{+} \mathrm{SR}$ down to $20 \mathrm{mK}$, constraining $\left|J_{\perp} / J\right|<6 \times 10^{-5}$, making this the most successful realization of a 1DQHAFM yet reported. In this case, the weak relaxation that is observed via $\mu^{+} \mathrm{SR}$ allows the investigation of the magnetic fluctuation spectrum. Whether the excitations in 1DQHAFs are ballistic or diffusive remains a key question in the physics of spin chains [26, 27] and one that can be addressed with LF $\mu^{+} \mathrm{SR}$ measurements. Field-dependent studies can be used to distinguish between these types of spin transport, since their spin autocorrelation functions have different associated spectral densities $f(\omega)$ which

vary as $\omega^{-\frac{1}{2}}$ for $d=1$ diffusive transport and $f(\omega) \propto \ln (J / \omega)$ for ballistic motion. In DEOCC-TCNQF 4 diffusive transport was identified. This contrasts with the inorganic 1DQHAF $\mathrm{Rb}_{4} \mathrm{Cu}\left(\mathrm{MoO}_{4}\right)_{3}$ where ballistic transport was shown to dominate the muon relaxation [28]. The details and general rules of the $d=1$ transport are complicated and this continues to be an area exciting much interest and new research.

\section{Two dimensions}

The model of the two-dimensional square lattice quantum Heisenberg antiferromagnet (2DSLQHA) has received significant attention as another apparently simple system 

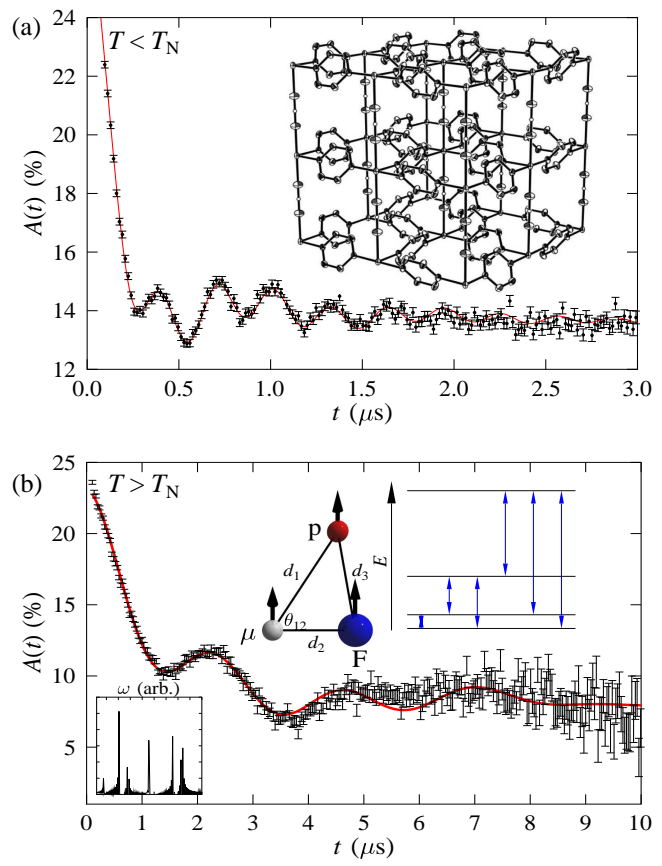

Figure 4. Zero field $\mu^{+} \mathrm{SR}$ data measured on $\left[\mathrm{CuHF}_{2}(\mathrm{pyz})_{2}\right]\left(\mathrm{BF}_{4}\right)$. (a) Zero field oscillations below $T_{\mathrm{N}}=1.54 \mathrm{~K}$ allow us to identify LRO and probe the critical behaviour. [32] (b) For $T>T_{\mathrm{N}}$ we observe muon-fluorine dipole-dipole oscillations that allow us to deduce muon sites [33, 34].

where the influence of dimensionality leads to the emergence of an unusual ground state and excitation spectrum [29]. It is also, of course, the low-energy model that describes the Mott insulating parent phase of the cuprate superconductors. The 2DSLQHA shows no magnetic order for $T>0$, owing to the Coleman-Mermin-Wagner theorem [30, 31], but there is good evidence that it orders at $T=0$ with a much renormalized ordered moment of around $0.62 \mu_{\mathrm{B}}[29]$. The excitations of the model at $T=0$ are gapless spin wave excitations with a linear dispersion [4, [5, 29].

Of the materials recently synthesised which show two-dimensional $(d=2)$ magnetic properties, one of the most successful classes is based on two-dimensional square layers of $\mathrm{Cu}^{2+}$ linked with pyrazine groups to form $\left[\mathrm{Cu}(\mathrm{pyz})_{2}\right]^{2+}$ layers with an intralayer exchange coupling $J$. These square layers may be linked with $\mathrm{HF}^{2-}$ ions to form pseudocubic arrays of $\left[\mathrm{CuHF}_{2}(\mathrm{pyz})_{2}\right] X$ [shown inset in Fig. 4(a) [32], where $X$ is an anion that sits in the centre of the cube and provides charge neutrality. As in the $d=1$ case, we are able to identify magnetic order in this system at low temperature and assess the degree to which it may be described as two-dimensional [33]. As an example we may consider $\left[\mathrm{CuHF}_{2}(\mathrm{pyz})_{2}\right] \mathrm{BF}_{4}$ (Fig. 4) which magnetically orders at $T=1.54 \mathrm{~K}$ [32]. If we attribute the ordering to an interlayer coupling $J_{\perp}$ we may use the results of quantum Monte Carlo calculations which, in this case, predict [22] that

$$
\left|J_{\perp} / J\right|=\exp \left(b-\frac{4 \pi \rho_{\mathrm{s}}}{T_{\mathrm{N}}},\right),
$$



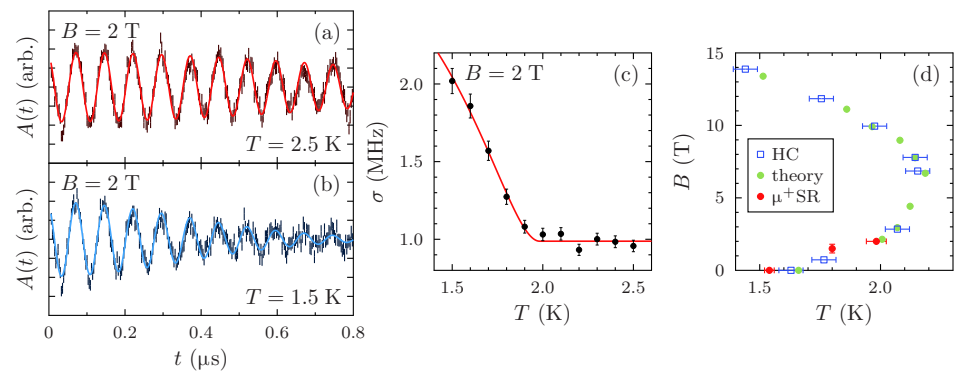

Figure 5. (a,b) Transverse field $\mu^{+} \mathrm{SR}$ measurements on $\left.\mathrm{Cu}\left(\mathrm{HF}_{2}\right)(\mathrm{pyz})_{2}\right] \mathrm{BF}_{4}$. (c) The relaxation rate allow us to extract the ordering temperature in field. (d) The result is a non-monotonic phase boundary caused by a small $X Y$-like component in the spin Hamiltonian of the system [33.

where $\rho_{\mathrm{s}}=0.183 J$ is the spin stiffness and the parameter $b=2.43$. For $\left[\mathrm{CuHF}_{2}(\mathrm{pyz})_{2}\right] \mathrm{BF}_{4}$ we find $J=6.3 \mathrm{~K}$ and $\left|J_{\perp} / J\right|=9 \times 10^{-4}$, making this material a highly anisotropic $d=2$ antiferromagnet.

In addition, the fact that these systems contain fluorine leads to oscillations at temperatures above $T_{\mathrm{N}}$ attributable to $\mathrm{F}-\mu^{+}$dipole-dipole coupling [Figure 4(b)]. These are useful here as they allow us to work out where the muons giving rise to these signals are localised with respect to the fluorine nuclei in these materials [34]. It is worth noting however that in complex materials such as these there are often several classes of muon sites, not all of which may be accounted for by those muons giving $\mathrm{F}-\mu^{+}$oscillations.

Although interlayer coupling in a 2DQHAF again provides a means for magnetic order to occur at non-zero temperatures there is evidence that in this system a degree of $g$-factor anisotropy causes the $\mathrm{Cu}^{2+}$ ions to take on a degree of $X Y$-like behavior [35]. This is of interest as the pure $X Y$-model allows the occurrence of a new sort of excitation: the vortex. A single vortex is unstable alone, and in the absence of an external electromagnetic field, we may only stabilise vortices at low temperature by binding them with antivortices. The transition between bound vortex-antivortex pairs and single vortices stabilised by thermal fluctuations at high temperature was originally identified by Kosterlitz and Thouless and by Berezinskii for an ideal system, but has never been observed in a bulk magnet [36, 37, 38]. However, the physics that those authors identified may have an effect even when only a small $X Y$-like component is present. In this case we expect that, while at high temperature the spins will be Heisenberg-like, as the temperature is lowered to a scale where the $X Y$-like component is resolvable it becomes energetically favourable for the spins to tip into the $X Y$-plane. On further decrease of temperature the possibility of a vortex-binding transition greatly increases the correlation length, at which point the interlayer coupling $J_{\perp}$ causes the system to undergo a transition to LRO [35].

One experimental consequence of $X Y$-like behaviour is that the phase boundary in applied magnetic field takes on a non-monotonic form as shown in figure 5] [39, 33]. 
The application of the field makes it energetically favourable for the spins to tip into the $X Y$ plane, effectively reducing the dimensionality. It then has a dual effect on

the spins by suppressing the amplitude of the order parameter by forcing spins along a particular direction and also reducing the phase space for phase fluctuations from a sphere to a circle. At low fields the magnetic ordering transition is controlled by phase fluctuations and at higher field by amplitude fluctuations leading to the non-monotonic phase boundary that is observed in experiment [39]. We have been able to measure the phase boundary in field using both $\mathrm{LF} \mu^{+} \mathrm{SR}$ and transverse field (TF) $\mu^{+} \mathrm{SR}$ [33] as shown for $\left[\mathrm{Cu}\left(\mathrm{HF}_{2}\right)(\mathrm{pyz})_{2}\right] \mathrm{BF}_{4}$ in figure 5, It has been argued [39] that the energy scales for this physics are controlled by the Kosterlitz-Thouless physics described above. It is probable that the physics of vortices, along more complicated topological objects such as merons and skyrmions, will be an area of future interest in this field.

\section{Switching the dimensionality}

We might ask if it is possible to gain control over the dimensionality of a system. There have been two recent advances that suggest that this is a fruitful area for future work: the first a synthetic route; the second using pressure as a means of creating a dimensionality switch.

The first route [40] relies on varying the proportions of reactants in the material synthesis, which makes it possible to create two distinct, but closely related materials, one two-dimensional; the other one-dimensional. The coordination polymer $\left[\mathrm{Cu}(\text { pyz })_{2}(\text { pyO })_{2}\right]\left(\mathrm{PF}_{6}\right)_{2}$ (where pyO denotes pyridine- $\mathrm{N}$-oxide $=\mathrm{C}_{5} \mathrm{H}_{5} \mathrm{NO}$ ) is another $d=2$ square lattice material based on $\left[\mathrm{Cu}(\text { pyz })_{2}\right]^{2+}$ layers, this time with pyO groups protruding from them. By changing the proportion of pyz and pyO molecules in the preparation from 3:1 to 2:1 the water-containing material $\left[\mathrm{Cu}(\mathrm{pyz})(\mathrm{pyO})\left(\mathrm{H}_{2} \mathrm{O}\right)_{2}\right]\left(\mathrm{PF}_{6}\right)_{2}$ is produced. Here pyz ligands link $\mathrm{Cu}$ atoms along the $b$-axis only, forming highly one-dimensional chains. Crucially, the strong nearest-neighbour exchange interaction maintains a value of $J \approx 8 \mathrm{~K}$ in both cases.

These structures suggest $d=2$ and $d=1$ magnetic behaviour, which is indeed confirmed by a combination of high-field magnetometry and muon-spin relaxation [40]. The results of the $\mu^{+} \mathrm{SR}$ measurements are shown in figure 6 where we are able to establish where each material undergoes a transition to LRO. The ordering temperature and magnetic moment size of the $d=2$ material are both larger than in the analogous $d=1$ material, which reflects the expected increase in the influence of fluctuations for $d=1$ compared to $d=2$. We conclude, in this case, that to turn a material from $d=2$ to $d=1$, one must just add water!

The second method of controlling dimensionality involves using pressure as a switch [20]. The material $\mathrm{CuF}_{2}\left(\mathrm{H}_{2} \mathrm{O}\right)_{2}$ pyz [41] is based on a chain-like structure but, rather counter-intuitively, it is a good realization of a 2DQHAFM. The reason for this is that the chains arrange themselves in such a way as to form square layers of $\mathrm{Cu}^{2+}$ ions with superexchange pathways provided by hydrogen bonds. Crucially the unfilled $d_{x^{2}-y^{2}}$ 

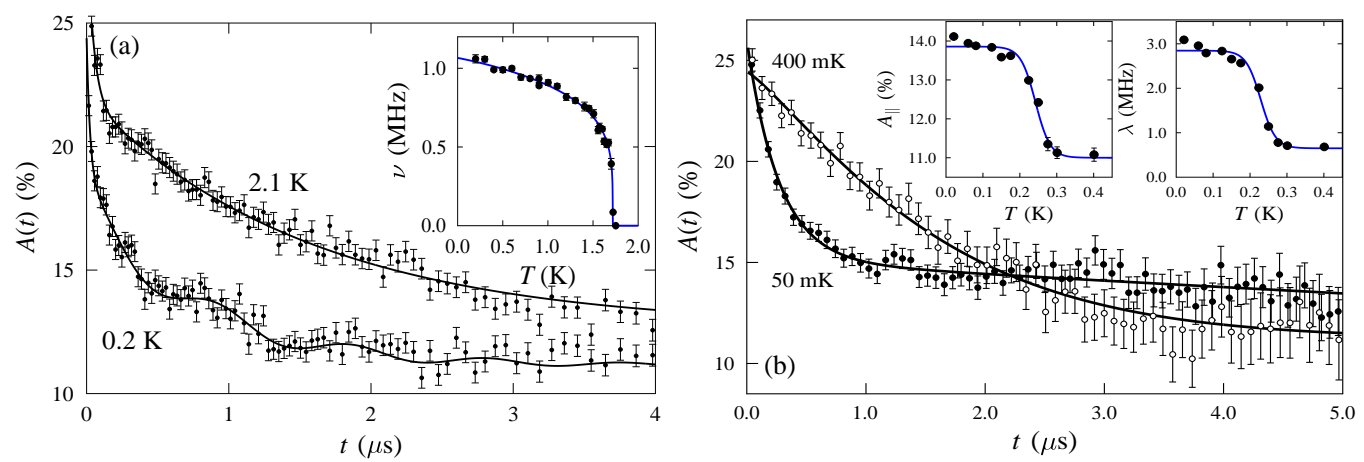

Figure 6. Dimensionality switching: $\mu^{+} \mathrm{SR}$ allows us to probe magnetic order in (a) $d=2\left[\mathrm{Cu}(\mathrm{pyz})_{2}(\mathrm{pyO})_{2}\right]\left(\mathrm{PF}_{6}\right)_{2}$ and $\left.(\mathrm{b}) d=1 \mathrm{Cu}(\mathrm{pyz})(\mathrm{pyO})\left(\mathrm{H}_{2} \mathrm{O}\right)_{2}\right]\left(\mathrm{PF}_{6}\right)_{2}$. [40]

orbital lies in the plane of the square layers, providing strong two-dimensional exchange with only weak coupling along the chains.

Applying hydrostatic pressure causes a dramatic change in behaviour above around $10 \mathrm{kbar}[20$. Here the magnetization shifts from that typical of a $d=2$ system to that of a $d=1$ magnet. $\mu^{+} \mathrm{SR}$ has been carried out under pressure and reveals the change in $T_{\mathrm{N}}$ as expected from a change from $d=2$ to $d=1$. The microscopic explanation for this quantum phase transition is suggested by a combination of x-ray diffraction and electron spin resonance [42, 43]. The application of large hydrostatic pressures causes a

small structural distortion which, in turn, causes the Jahn-Teller axis of the $\mathrm{Cu}^{2+}$ ions to switch directions, resulting in the $d_{x^{2}-y^{2}}$ orbital shifting from linking $\mathrm{Cu}$ atoms in hydrogen bonded $\left[\mathrm{Cu}(\text { pyz })_{2}\right]$ layers to point along the $\mathrm{Cu}$-pyz- $\mathrm{Cu}$ chains.

It is possible that such a switching of magnetic dimensionality will be found, and ultimately engineered, in a variety of magnetic coordination polymers. The fundamental change in the character of a system in going from $d=2$ to $d=1$ is such that this switching feature could conceivably find a use in future quantum technologies.

\section{Frontiers: a zoology of ground states}

We now turn to a possible future direction for the engineering of coordination polymers. We hope that it will soon be possible to produce materials that realise still more complicated models of low-dimensional magnetic behaviour. These would allow us to realise a large number of new and potentially exotic ground states. Here we briefly review the predictions for some of the quantum states that may be achieved if we are able to tune the interaction strength of our $s=1 / 2$ spins. For the full story we refer you to some of the excellent books on this subject [4, [5, 6].

\section{1. $d=1$ : Tomonaga-Luttinger, Valence bond solid and Ising-Néel states}

When describing the 1DQHAFM in section 2 we claimed that the disordered T-L ground state with its gapless linear excitation spectrum was reached if the coupling was not 
too large. More precisely, we mean that that the antiferromagnetic nearest neighbour exchange coupling $J_{1}$ is dominant and the antiferromagnetic next-nearest neighbour exchange $J_{2}$ is small in comparison. However, if the ratio of the couplings exceeds a critical value $J_{2} / J_{2}>J_{\mathrm{c}}$ then the system adopts another ground state: the spin-Peierls or (in more modern terminology) the valence bond solid (VBS) state, illustrated in figure 2(iii). The VBS ground state involves the spins staggering their bond exchange energy, essentially forming antiferromagnetically coupled dimers along the chain. There are two ways of doing this along any chain (since a particular site may form a dimer with its neighbour to the left, or to the right) and so the ground state that is realised must break translational symmetry. An antiferromagnetically coupled dimer has a singlet ground state, resulting in a total $S=0$ ground state for the VBS. The excitations of the VBS phase are lone spins sat between the valence bonds. These are spin $s=1 / 2$ excitations which, as in the T-L case are also known as spinons but here there is an energy gap $\Delta$ which must be exceeded to create such an excitation. In fact, the energy required to excite a spinon of momentum $\boldsymbol{p}$ is given by $E_{\boldsymbol{p}}=\left(\Delta^{2}+v_{\mathrm{F}}^{2} \boldsymbol{p}^{2}\right)^{\frac{1}{2}}$, where $v_{\mathrm{F}}$ is the Fermi energy of the system.

Although we have hitherto concentrated only on low-energy models which include purely spin degrees of freedom, the realization of the VBS state also requires the inclusion of magnetoelastic coupling (and it is in this context that it is usually called a spin-Peierls state). Above the spin-Peierls transition temperature $T_{\mathrm{SP}}$, we have a uniform antiferromagnetic next-neighbour exchange in each chain; below $T_{\mathrm{SP}}$ there is an elastic distortion resulting in the dimerization described abve, and hence two, unequal, alternating exchange constants. The alternating chain possesses an energy gap between the singlet ground state and the lowest lying band of triplet excited states which closes up above $T_{\mathrm{SP}}$. The transition temperatures may be related to the relevant coupling constants; whereas the conventional Peierls distortion is expected at a temperature $T_{\mathrm{P}} \sim\left(E_{\mathrm{F}} / k_{\mathrm{B}}\right) \exp (-1 / \Lambda)$, where $E_{\mathrm{F}}$ is the Fermi energy of the system and $\Lambda$ is the electron-phonon coupling constant, the spin-Peierls transition is expected at $T_{\mathrm{sP}} \sim\left(J / k_{\mathrm{B}}\right) \exp (-1 / \Lambda) . J \ll E_{\mathrm{F}}$ and hence $T_{\mathrm{sP}} \ll T_{\mathrm{P}}$ [44].

One example system is the organic salt $\mathrm{MEM}(\mathrm{TCNQ})_{2}$, which consists of onedimensional stacks of planar TCNQ molecules, each of which has a charge of $-\frac{1}{2} e$ associated with it. Adjacent stacks are separated by arrangements of MEM molecules, each of which possess a localized charge of $+e$. It undergoes two structural distortions. The first, which occurs at $335 \mathrm{~K}$, is a conventional Peierls transition in which the TCNQ chains dimerize. This results in a change from metallic to insulating behaviour as a single electronic charge becomes localized on each TCNQ dimer; the single spin on each dimer couples antiferromagnetically to its neighbours. This phase persists down to the spin-Peierls transition at $18 \mathrm{~K}$, where a further dimerization of the TCNQ stacks takes place (this is a tetramerization of the original chain). $\mu \mathrm{SR}$ studies indicate a slowing down of the electronic fluctuations resulting from the opening of the spin-Peierls gap in the magnetic excitation spectrum as the temperature is lowered below $T_{\mathrm{sP}}[45]$. At the very lowest temperatures the electronic spin fluctuations freeze out and the muon- 
spin depolarization is dominated by persistent slow fluctuations which can be ascribed to a defect-spin system [46]. It has been speculated that the muon spin might locally create a spin defect by breaking a singlet pair, giving rise to the relaxation ascribed to defect spins and which is only revealed when the other sources of relaxation have frozen out [46]. These results have now been extended to the related material DEM(TCNQ $)_{2}$ [47. Other examples of spin-Peierls systems include other organic systems such as $\mathrm{TTF}-\mathrm{CuS} \mathrm{C}_{4}\left(\mathrm{CF}_{3}\right)_{4}\left(T_{\mathrm{sP}}=12 \mathrm{~K}\right)$ [48] and $\mathrm{TTF}-\mathrm{AuS}_{4} \mathrm{C}_{4}\left(\mathrm{CF}_{3}\right)_{4}\left(T_{\mathrm{sP}}=2 \mathrm{~K}\right)$ [48, 49]. A common feature of such materials is flat organic molecules in columnar stacks. The large interchain separation and weak van der Waals intermolecular interactions favour the dominance of magnetoelastic effects over interchain ordering. In contrast the chains in corresponding inorganic materials, such as copper chain compounds, are quite rigid due to the ionic bonding and only a single example of an inorganic spin-Peierls material is known $\left(\mathrm{CuGeO}_{3}\right.$, with $\left.T_{\mathrm{sP}}=14 \mathrm{~K}[50]\right)$.

As the previous example of the VBS illustrates, allowing strong interactions from distant spins opens up new possibilities of interesting phases to study. In fact a rich seam of physics is revealed if start with a $d=1$ spin Hamiltonian, and allow the possibility of both an $X Y$-like spin anisotropy component and next-nearest neighbour interactions. This is the content of the $J_{1}-J_{2}$ model, whose Hamiltonian is given by

$$
\hat{H}=J_{1} \sum_{\langle i j\rangle}\left(\hat{S}_{i}^{x} \hat{S}_{j}^{x}+\hat{S}_{i}^{y} \hat{S}_{j}^{y}+\alpha \hat{S}_{i}^{z} \hat{S}_{j}^{z}\right)+J_{2} \sum_{i k}\left(\hat{S}_{i}^{x} \hat{S}_{k}^{x}+\hat{S}_{i}^{y} \hat{S}_{k}^{y}+\alpha \hat{S}_{i}^{z} \hat{S}_{k}^{z}\right),
$$

where $i$ and $j$ label nearest-neighbours; $i$ and $k$ label next-nearest neighbours and $\alpha$ is a parameter which we may vary from 0 ( $X Y$ spins), through 1 (Heisenberg spins) to large values (resulting in Ising spins). This model may, in turn, be mapped onto the sine-Gordon model of quantum field theory [5, 51] (the origin of the latter's curious name is described in Ref [51]) and whose RG scaling analysis leads to the phase diagram shown in figure 2(ii).

From figure 2(ii) we see that tuning the parameters of this model allows us to access three ground states: the T-L liquid; the VBS (both described above) and the Ising-Néel state. [It is worth noting that along the line $\alpha=1$ (corresponding to Heisenberg spins) it can be argued that for $J_{2} / J_{1}<J_{c}$ the system is a T-L liquid but for $J_{2} / J_{1}>J_{c}$ the system adopts the VBS state (as claimed above and in section 2)]. The Ising-Néel state, reached by making $\alpha>1$, with moderate next-nearest neighbour coupling, is characterized by a spontaneously broken symmetry at $T=0$. Here we have magnetic LRO (not seen in either the T-L or VBS states) involving a staggered expectation value for the $z$-component of the spins. The lowest energy excitations of this phase are gapped $s=1 / 2$ spinons. The properties of these states are summarised in table 1 .

\section{2. $d=2$ : quantum criticality and spin liquids}

The ground state for the 2DQHAF is antiferromagnetically (Néel) ordered at $T=0$ [4, 5, 29]. This phase has gapless spin-wave excitations which do not carry a definite spin. (However, if the Néel order is aligned along $z$, the spin waves have total $S_{z}= \pm 1$.) 


\begin{tabular}{cccc}
\hline Phase & $T=0$ ground state & excitation & character \\
\hline Tomonaga-Luttinger liquid & Spin disordered & $s=1 / 2$ spinons & gapless \\
Valence bond solid & Dimerised & spinons & gapped \\
Ising-Néel & Néel ordered & spinons & gapped \\
\hline
\end{tabular}

Table 1. Phases of the $d=1$ magnet.

\begin{tabular}{cccc}
\hline Phase & $T=0$ ground state & excitation & character \\
\hline Néel $\left(g<g_{\mathrm{c}}\right)$ & Staggered order & Total $S_{z}=1$ spin waves & gapless \\
Quantum paramagnet $\left(g>g_{\mathrm{c}}\right)$ & disordered & $s=1$ flipped spins & gapped \\
Valence bond solid $\left(g>g_{\mathrm{c}}\right)$ & total $S=0$ & $s=1$ & gapped \\
\hline
\end{tabular}

Table 2. Phases of the $d=2$ Heisenberg magnet. The inclusion of Berry phases in the theoretical description leads to the VBS phase for $g>g_{\mathrm{c}}$.

As we increase the strength of the effective long-range coupling (which we call $g$ here) another phase is realised above a QCP at a critical effective coupling $g_{c}$, as shown in figure 2(iv). This phase, which is often called a quantum paramagnet, does not show LRO and has a gap against $s=1$ excitations, which may be thought of as flipped spins. This state can also be realised in the sort of spin model we have been considering by including next-nearest neighbour interactions as we discussed for the $d=1$ model. In fact, numerical and series expansion studies suggest that $g_{\mathrm{c}}$ corresponds to a ratio between next-nearest and nearest neighbours of $J_{2} / J_{1}=J_{\mathrm{c}} \approx 0.4$. The arguments leading to the quantum paramagnet neglect the contribution of Berry phases to the final configuration of the system. If these are included a VBS state is realised with singlet bonds lining up in columns or plaquettes on the $d=2$ square lattice. Such a ground state is four-fold degenerate and so, as in the $d=1$ case, the VBS state that is realised must break translational symmetry. However, the lowest lying excitation is the same as predicted for the quantum paramagnet (a gapped $s=1$ quasiparticle), but higher excitations will be different [5].

Finally, although everything we have discussed in this section applied for $T=0$, we may consider non-zero temperatures in this $d=2$ case, which results in the phase diagram shown in figure 2(iv). The curved lines represent crossovers in behavior which allow us to identify a quantum critical region, which extends down to $T=0$ for $g=g_{c}$. Here, both quantum fluctuations (i.e. time dependence in the wavefunctions deriving from uncertainty relations) and thermal fluctuations are equally important and it is impossible to describe the excitations in terms of nearly-free quasiparticles. In this region the phase of the wavefunctions are as incoherent as Nature allows, realising an (almost) ideal fluid. Quantum critical behaviour continues to be an area of much experimental and theoretical interest. The properties of the $d=2$ system are summarised in table 2

As an example of how muons may be useful in elucidating the physics of the exotic phases described here, we mention the case of $\kappa$-(BEDT-TTF $)_{2} \mathrm{Cu}_{2}(\mathrm{CN})_{3}$ [52]. This 
(a)

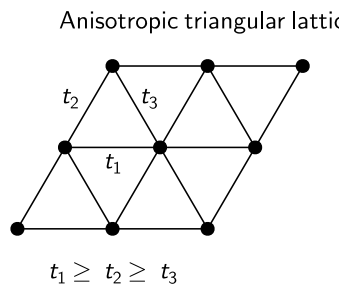

(d)

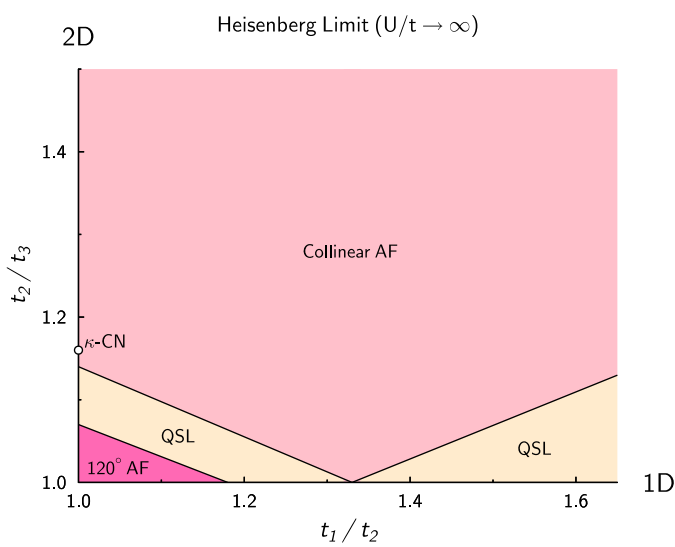

(b)

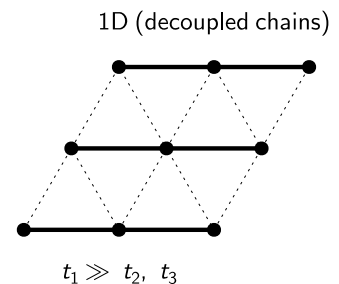

(c)

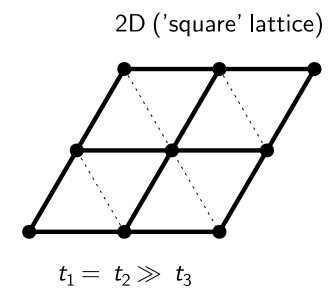

(e)

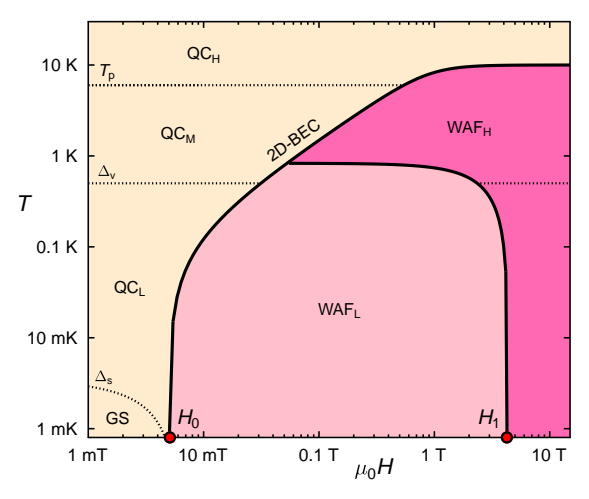

Figure 7. (a) The triangular lattice with couplings $t_{1}, t_{2}$ and $t_{3}$ as shown. By varying the coupling we may realize (b) the $d=1$ or (c) the $d=2$ cases. (d) Generalized phase diagram 53 for the triangular lattuce. (QSL=quantum spin liquid phase, $\kappa$ - $\mathrm{CN}=\kappa$ $(\mathrm{BEDT}-\mathrm{TTF})_{2} \mathrm{Cu}_{2}(\mathrm{CN})_{3}$.) (e) Phase diagram of $\kappa$-(BEDT-TTF $)_{2} \mathrm{Cu}_{2}(\mathrm{CN})_{3}$ derived from $\mu^{+} \mathrm{SR}$ measurements [52].

is a $d=2$ system based around a triangular lattice of (antiferromagnetically coupled) $s=1 / 2$ spins, whose low energy physics provides an example of a frustrated, noncollinear antiferromagnet. In fact, the triangular lattice, shown in figure 7(a) may be viewed as a meeting point of the $d=1$ and $d=2$ cases. We may recover those cases by tuning the coupling constants to form either well coupled $d=1$ chains (e.g. by taking $\left.t_{1} \gg t_{2}, t_{3}\right)$ [figure 7(b)] or, with the choice $t_{1}=t_{2} \gg t_{3}$, forming a lattice which is topologically equivalent to the $d=2$ square lattice case [figure 7(c)]. A general phase diagram for the triangular lattice, derived from calculations using modified spin wave theory [53] and assuming perfectly localized Heisenberg spins, is shown in Fig. 77(d). The $d=1$ case is realized far along the horizontal axis, where the T-L ground state is identified as one example of a quantum spin liquid (QSL) state (here used as a general term used to describe a $T=0$ ground state without LRO, of which there are potentially very many varieties). The $d=2$ case is found far along the vertical axis showing the expected $T=0$ collinear (Néel) LRO. Of note in figure 7(d) is the isotropically coupled case $\left(t_{1}=t_{2}=t_{3}\right)$ where the classical solution of antiferromagnetic LRO with a $120^{\circ}$ spin structure is realised, albeit with moments much reduced by quantum fluctuation. This neighbours a QSL state for the case where the couplings are slightly anisotropic. 
Returning to the case of $\kappa$-(BEDT-TTF $)_{2} \mathrm{Cu}_{2}(\mathrm{CN})_{3}$, we see that its couplings appear to place it on the border of the QSL and Néel phases in figure7(d). In fact, a more realistic description of the material is acheived by assuming that $U / t$ (the ratio of the electronic correlation energy to the bandwidth) is finite. This, which amounts physically to a relaxation of the assumption that the relevant electronic degrees of freedom are perfect Heisenberg spins (or so-called "quantum tops") is expected to destabilise phases showing LRO near the origin in figure 7(d), enlarging the QSL phase to such an extent that $\kappa$-(BEDT-TTF $)_{2} \mathrm{Cu}_{2}(\mathrm{CN})_{3}$ should be expected to realize a QSL ground state. In fact, the $\mu^{+}$SR measurements [52] suggest that the material realizes a $Z_{2}$ QSL ground state showing topological order [5], with total $S=0$ and $s=1 / 2$ spinon excitations with a very small gap against their creation. (The " $Z_{2}$ " assignment tells us that the system is described by a topological gauge field theory [51] somewhat similar to those used to describe fractional quantum Hall fluids, whose description is locally invariant under the $Z_{2}$ group of transformations of the fields.) There is also evidence for another type of gapped excitation in this system, which is a point-like vortex known as a vison. For $\kappa$-(BEDT-TTF $)_{2} \mathrm{Cu}_{2}(\mathrm{CN})_{3}$ muon measurements [52] not only suggest this complex excitation spectrum, but the application of magnetic fields allows the system to be driven into other phases showing LRO with extremely small magnetic moments, leading to the phase diagram shown in figure 7(e). The exquisite sensitivity of $\mu^{+} \mathrm{SR}$ and the potential to apply it to samples which are smaller than those required for inelastic neutron measurements have made it particularly useful in this context.

\section{Conclusion}

In the short term muons will continue to allow us to probe low-moment LRO and aspects of the excitation spectrum in low-dimensional magnetic systems based on molecular building blocks. In addition to the cases outlined here there are many other avenues for future study in this field, including the investigation of $d=0$ systems such as those based on dimers or on molecular nanomagnets formed from clusters containing large numbers of strongly coupled spins. Also of interest are spin-ladder systems which interpolate between the harsh extremes of $d=1$ and $d=2$. In the future, the possibility of being able to work in more extreme conditions involving large applied magnetic fields and pressures will open out more of the phase diagrams of these systems. At the same time, advances in materials synthesis will allow us to approach the platonic ideals of the models described in this Comment.

\section{Acknowledgments}

We would like to express particular thanks to J L Manson, J A Schlueter and C P Landee for their long-term collaboration on this work. We are also most grateful to all of our collaborators and students and to the staff of ISIS and PSI muon facilities. We thank P Hauke for providing modified spin wave theory results and P A Goddard and 
F Xiao for useful discussions regarding this Comment. TL is supported by EPSRC.

\section{References}

[1] Blundell S J 2007 Contemp. Phys. 48275

[2] Blundell S J and Pratt F L 2004 J. Phys.: Condens. Matter 16 R771

[3] Blundell S J 1999 Contemp. Phys. 40175

[4] Tsvelik A M 2003 Quantum Field Theory in Condensed Matter Physics, second edition (Cambridge: CUP)

[5] Sachdev S2011 Quantum Phase Transitions, second edition (Cambridge: CUP)

[6] Giamarchi T 2003 Quantum physics in one dimension (Oxford: OUP)

[7] Wilson K G 1975 Rev. Mod. Phys. 47773

[8] Brezin E and Zinn-Justin J 1976 Phys. Rev. Lett. 36691

[9] Blundell S J, Pratt F L, Marshall I M, Steer C A, Hayes W, Letard J F, Heath S L, Caneschi A and Gatteschi D 2003 Synthetic Metals 133531

[10] Pratt F, Lancaster T, Baker P, Blundell S J, Kaneko W, Ohba M, Kitagawa S, Ohira-Kawamura S and Takagi S, 2009 Physica B 404585

[11] Blundell S J, Lancaster T, Pratt F L, Baker P J, Brooks M L, Baines C, Manson J L and Landee C P 2007 J. Phys. Chem. Solids 682039

[12] Blundell S J, Lancaster T, Brooks M L, Pratt F L, Coronado E, Galan-Mascaros J R, Manson J L, Cadiou C and Winpenny R E 2005 Synthetic metals 152481

[13] Blundell S J, Pratt F L, Lancaster T, Marshall I M, Steer C A, Heath S L, Letard J F, Sugano T, Mihailovic D and Omerzu A 2003 Polyhedron 221973

[14] Blundell S J, Pattenden P A, Pratt F L, Valladares R M, Sugano T and Hayes W 1995 Europhys. Lett. 31573

[15] Lascialfari A, Gastteschi D, Borsa F, Shastri A, Jang Z H and Carretta P 1998 Phys. Rev. B 57 514

[16] Blundell S J2001 Magnetism in Condensed Matter (Oxford: OUP)

[17] We use the single- $J$ convention here where the energy gap between the singlet and triplet level of a two spin system with Hamiltonian $\hat{H}=J \boldsymbol{S}_{i} \cdot \boldsymbol{S}_{j}$ is given by $J$.

[18] Sengupta P, Sandvik A W, and Singh R R P 2003 Phys. Rev. B 68094423

[19] Goddard P A, Singleton, J Maitland C, Blundell S J, Lancaster T, Baker P J, McDonald R D, Cox S, Sengupta P, Manson J L, Funk K A and Schlueter J A 2008 Phys. Rev. B 78052408

[20] Ghannadzadeh S et al. arXiv:1304.5403 (2013).

[21] Lancaster T, Blundell S J, Brooks M L, Baker P J , Pratt F L, Manson J L, Landee C P and Baines C 2006 Phys. Rev. B 73 020410(R)

[22] Yasuda C, Todo S, Hukushima K, Alet F, Keller M, Troyer M, and Takayams H 2005 Phys. Rev. Lett. 94217201

[23] Schulz H J 1996 Phys. Rev. Lett. 772790

[24] Goddard P A, Singleton J, Sengupta P, McDonald R D, Lancaster T, Blundell S J, Pratt F L, Cox S, Harrison N, Manson J L, Southerland H I and Schlueter J A 2008 New J. Phys. 10083025

[25] Pratt F L, Blundell S J, Lancaster T, Baines C, and Takagi S 2006 Phys. Rev. Lett. 96247203

[26] Sirker J, Pereira R G and Affleck I 2009 Phys. Rev. Lett 103216602

[27] Steinigeweg R and Brenig W 2011 Phys. Rev. Lett. 107250602

[28] Lancaster T, Baker P J, Pratt F L,Blundell S J,Hayes W and Prabhakaran D 2012 Phys. Rev. B 85184404

[29] Manousakis E 1991 Rev. Mod. Phys. 631

[30] Mermin N D and Wagner H 1966 Phys. Rev. Lett. 171133

[31] Coleman S 1973 Comm. Math. Phys. 31259

[32] Manson J L, Conner M M, Schlueter J A, Lancaster T, Blundell S J, Brooks M L, Pratt F L, Papageorgiou T, Bianchi A D, Wosnitza J and Whangbo M-H 2006 Chem. Commun. 4894 
[33] Steele A J, Lancaster T, Blundell S J, Baker P J, Pratt F L, Baines C, Conner M M, Southerland H I, Manson J L and Schlueter J A 2011 Phys. Rev. B 84064412

[34] Lancaster T, Blundell S J, Baker P J, Hayes W, Pratt F L, Manson J L, Conner M M Schlueter J A 2007 Phys. Rev. Lett 99267601

[35] Xiao F, Woodward F M, Landee C P, Turnbull M M, Mielke C and Harrison N, Lancaster T, Blundell S J, Baker P J, Babkevich P and Pratt F L 2009 Phys. Rev. B 79134412

[36] Berezinskii V L 1971 Sov. Phys. JETP 32493

[37] Kosterlitz J M and Thouless D J 1973 J. Phys. C 61181

[38] Bramwell S T and Holdsworth P C W 1994 Phys. Rev. B 498811

[39] Sengupta P, Batista C D, McDonald R D, Cox S, Singleton, J Huang L, Papageorgiou T P, Ignatchik O, Herrmannsdörfer T, Manson J L, Schlueter J A, Funk K A, and Wosnitza J, 2009 Phys. Rev. B $\mathbf{7 9} 060409$

[40] Goddard P A, Manson J L, Singleton J, Franke I, Lancaster T, Steele A J, Blundell S J, Baines C, Pratt F L, McDonald R D, Ayala-Valenzuela O E, Corbey J F, Southerland H I, Sengupta $\mathrm{P}$ and Schlueter J A 2012 Phys. Rev. Lett. 108077208

[41] Manson J L, Conner M M, Schleuter J A, McConnell A C, Southerland H I, Malfant I, Lancaster T, Blundell S J, Brooks M L, Pratt F L, Singleton J, McDonald R D, Lee C, and Whangbo M-H 2008 Chem. Mater. 207408

[42] Halder G J, Chapman K W, Schlueter J A and Manson J L, 2011 Angew. Chem. Int. Ed. 50419

[43] Prescimone A, Monien C, Allan C, Schlueter J A, Tozer S, Manson J L, Parsons S, Brechin E K and Hill S 2012 Angew. Chem. Int. Ed. 517490

[44] Bray J W, Interrante L V, Jacobs I S and Bonner J C 1983 Extended Linear Chain Compounds, (Plenum, New York, 1983), 3353

[45] Blundell S J, Pratt F L, Pattenden P A, Kurmoo M, Chow K H, Jestädt T and Hayes W 1997 J. Phys.: Condens. Matter 9 L119

[46] Lovett B W, Blundell S J, Pratt F L, Jestadt T, Hayes W, Tagaki S and Kurmoo M 2000 Phys. Rev. B 6112241

[47] Blundell S J, Marshall I M, Lovett B W, Pratt F L, Husmann A, Hayes W, Takagi S and Sugano T 2001 Hyp. Int. 133169

[48] Bray J W, Hart H R, Interrante L V, Jacobs I S, Kasper J S, Watkins G D, Wee S H and Bonner J C 1975 Phys. Rev. Lett. 35744

[49] Jacobs I S, Bray J W, Hart H R, Interrante L V, Kasper J S, Watkins G D, Prober D E and Bonner J C 1976 Phys. Rev. B 143036

[50] Hase M, Terasaki I and Uchinokura K 1993 Phys. Rev. Lett. 703651

[51] Coleman S 1985 Aspects of Symmetry (Cambridge CUP)

[52] Pratt F L, Baker P J, Blundell S J, Lancaster T, Ohira-Kawamura S, Baines C, Shimizu Y, Kanoda K, Watanabe I and Saito G 2011 Nature 471612

[53] Hauke P 2013 Phys. Rev. B 87014415 